Review

\title{
Advances in the Function and Regulation of Hydrogenase in the Cyanobacterium Synechocystis PCC6803
}

\section{Corinne Cassier-Chauvat, Théo Veaudor and Franck Chauvat *}

UMR8221, CEA, CNRS, University of Paris XI, Institute of Biology and Technology Saclay, Laboratory of Biology and Biotechnology of Cyanobacteria, CEA-Saclay, Gif sur Yvette 91 191, France; E-Mails: corinne.cassier-chauvat@cea.fr (C.C.-C.); theo.veaudor@cea.fr (T.V.)

* Author to whom correspondence should be addressed; E-Mail: franck.chauvat@cea.fr; Tel.: +33-1-69-08-35-74.

External Editor: Patrick Hallenbeck

Received: 11 September 2014; in revised form: 15 October 2014 / Accepted: 21 October 2014 / Published: 31 October 2014

\begin{abstract}
In order to use cyanobacteria for the biological production of hydrogen, it is important to thoroughly study the function and the regulation of the hydrogen-production machine in order to better understand its role in the global cell metabolism and identify bottlenecks limiting $\mathrm{H}_{2}$ production. Most of the recent advances in our understanding of the bidirectional [Ni-Fe] hydrogenase (Hox) came from investigations performed in the widely-used model cyanobacterium Synechocystis PCC6803 where Hox is the sole enzyme capable of combining electrons with protons to produce $\mathrm{H}_{2}$ under specific conditions. Recent findings suggested that the Hox enzyme can receive electrons from not only $\mathrm{NAD}(\mathrm{P}) \mathrm{H}$ as usually shown, but also, or even preferentially, from ferredoxin. Furthermore, plasmid-encoded functions and glutathionylation (the formation of a mixed-disulfide between the cysteines residues of a protein and the cysteine residue of glutathione) are proposed as possible new players in the function and regulation of hydrogen production.
\end{abstract}

Keywords: hydrogen; bioproduction; cyanobacteria; oxidative stress; regulation; cysteine oxidation; sugar stress; electron transport; ferredoxin 


\section{Introduction}

Energy is crucial to modern industry. In 2012, 570 ExaJoules ( $1 \mathrm{EJ}=10^{18}$ Joules) of energy was consumed worldwide, of which approximately $80 \%$ was generated from burning fossil fuels thereby liberating into the atmosphere about 6 gigatons $\left(6 \times 10^{9}\right.$ tons $)$ of the green-house-generating gas $\mathrm{CO}_{2}$. Even hydrogen $\left(\mathrm{H}_{2}\right)$, which has a higher energy content than oil $\left(142 \mathrm{MJ} / \mathrm{kg}\right.$ for $\mathrm{H}_{2} v s .44 .2 \mathrm{MJ} / \mathrm{kg}$ for oil) and burns cleanly, producing only water as its by-product, is not yet a clean biofuel because it is mostly produced from burning oil [1]. Hence, the pollution problem and the fossil fuel shortfall anticipated to occur during the 21 st century make it important to develop new energy sources that are plentiful, renewable and environmentally friendly. Sunlight is naturally attractive as it is the most readily available and inexpensive source of energy on Earth. For instance, the annual solar flux received by Earth-approximately 5 YottaJoules ( $1 \mathrm{YJ}=10^{24}$ Joules $)$ - is in huge excess of the 570 ExaJoules used by our society. Consequently, there is a growing interest in processes that could couple the solar energy-powered capture of $\mathrm{CO}_{2}$ to the production of energies through the use of photosynthetic organisms, such as microalgae [2,3]. Indeed, production by microalgae of renewable biofuels from nature's most plentiful resources, solar light, water, mineral salts and $\mathrm{CO}_{2}$, is of great interest in recycling $\mathrm{CO}_{2}$ and saving arable soils, fertilizers, pesticides and fresh waters for crop production. Cyanobacteria (formerly termed blue-green algae) have the potential for that. First, cyanobacteria are the most abundant photosynthetic organisms of our planet. They colonize most ecological niches, fresh and salt waters, terrestrial and extreme environments $(\mathrm{pH}$, temperature) that counter-select most competing organisms, a primary concern of mass cultivation [4]. Second, cyanobacteria convert captured solar energy into biomass at high efficiencies (3\%-9\%) [2,5] to produce a large part of the atmospheric oxygen and organic assimilates for the food chain [6]. On a global scale, cyanobacteria fix an estimated 25 Giga tons of carbon from $\mathrm{CO}_{2}$ per year into energy dense biomass [7]. To perform this huge $\mathrm{CO}_{2}$ fixation, cyanobacteria use $0.2 \%-0.3 \%$ of the solar energy, 178,000 terawatts ( $1 \mathrm{TW}=10^{12}$ watts), reaching the Earth surface [8]. Thus, the amount of energy passing through cyanobacteria exceeds by more than 25 times the energy demand of our society; roughly 1000-fold the total nuclear energy produced on Earth. Third, as cyanobacteria tolerate high $\mathrm{CO}_{2}$ content in gas streams and they can grow in a variety of locations, they can be used as "low-cost" microbial cell factories for the capture and storage of industrial $\mathrm{CO}_{2}$ gas near the sites of industrial productions, thereby reducing transportation costs. Fourth, many cyanobacteria as the unicellular model strain Synechocystis PCC6803 have a small sequenced genome amenable to genetic manipulations including with versatile plasmid vectors [9-11]. A powerful genetics is welcome as natural (wild-type) cyanobacteria are not suitable biofuel producers because some of the required metabolic pathways are partially lacking or need optimization $[3,5]$.

In cyanobacteria, two enzymes can produce hydrogen, the [Ni-Fe] bidirectional hydrogenase (Hox for hydrogenase oxidation) and the nitrogenase. Both enzymes are sensitive to oxygen and do not occur in all strains, but for $\mathrm{H}_{2}$ production the [Ni-Fe] hydrogenase, which does not use ATP [12], is energetically favored over the nitrogenase enzyme that consumes 16 ATP per molecules of $\mathrm{H}_{2}$ produced [13].

The bidirectional Hox enzyme has been mostly studied in the model cyanobacterium Synechocystis PCC6803 (hereafter Synechocystis), where it is the sole enzyme capable of combining electrons 
with protons to produce $\mathrm{H}_{2}$ under specific conditions [12,14]. The Hox enzyme comprise five protein-subunits, HoxEFUYH, and a $[\mathrm{Ni}-\mathrm{Fe}]$ and several [Fe-S] redox clusters (Figure 1).

Figure 1. Schematic representation of hydrogen production machine in Synechocystis adapted from $[12,15]$. The genes are represented by arrows, which point in the direction of their transcription, and are colored similarly to their protein products. The green numbers indicate the spacing distance (in kilobases) between the scattered genes. The hoxEFUHY operon is weakly transcribed [16] as the polycistronic mRNA (bent blue arrow), which encodes (i) the hydrogenase sub-complex (made by the HoxY protein and the HoxW-matured HoxH subunit); (ii) the HoxEFU diaphorase sub-complex; and (iii) the three proteins of unknown function (white forms). The electron transfer FMN cofactor, Fe-Ni center, and $[4 \mathrm{Fe}-4 \mathrm{~S}]$ and $[2 \mathrm{Fe}-2 \mathrm{~S}]$ clusters of the Hox proteins, are represented by the blue squares, the pink star, dark-red squares and light-red diamonds, respectively. The zinc-bound to HypA1 and HypB1 proteins is shown as the blue form. CP designates carbamoyl phosphate. The brown lines stand for the reversible inactivation of Hox activity mediated by oxygen. The photosynthetic membrane is represented in green.

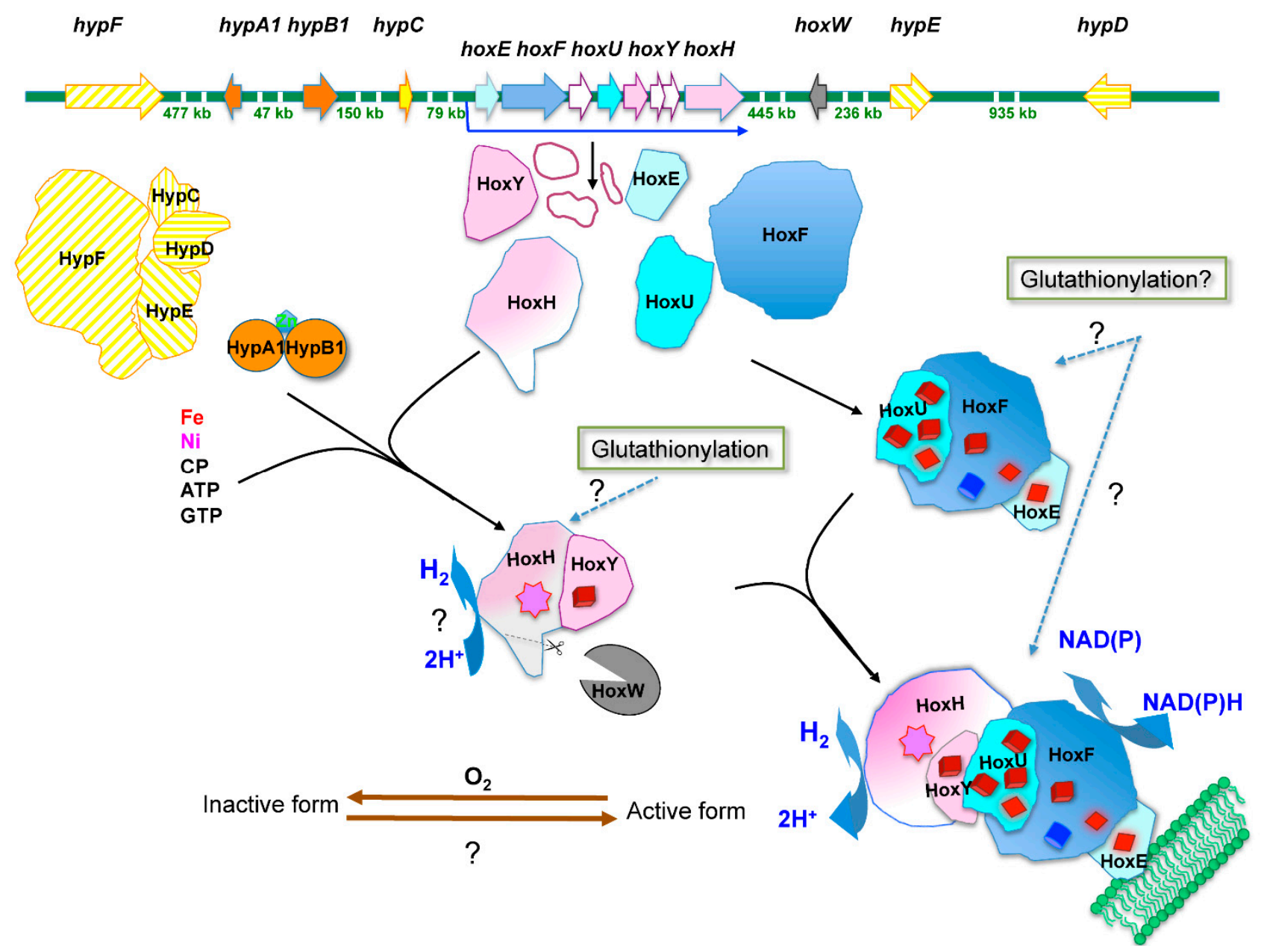


The HoxEFU subunits make up the diaphorase sub-complex that transfers to the [Ni-Fe] hydrogenase sub-complex HoxHY the NAD $(\mathrm{P}) \mathrm{H}$-transported electrons produced by photosynthesis and/or sugar catabolism [17]. Recently, based on an in vitro analysis, it was also proposed that the Hox enzyme can be reduced by ferredoxin or flavodoxin [18], similarly to the [Fe-Fe] hydrogenase of eukaryotic algae [19]. During HoxHY assembly, the HoxH subunit is processed by the HoxW protease [14], and subsequently the [Ni-Fe] HoxEFUYH complex is assembled by the six subunits HypABCDEF complex [12,14]. The Hox enzyme is dispensable to the photoautotrophic growth in standard laboratory conditions [20]; it has a bias towards $\mathrm{H}_{2}$ production [21] and it is reversibly inactivated by oxygen [17]. Hence, investigating the photobiological production of $\mathrm{H}_{2}$ by cyanobacteria has both an evident biotechnological interest, and a basic research interest, in addressing the paradox of the antagonistic production of hydrogen and oxygen $\left(\mathrm{O}_{2}\right.$ inhibits $\mathrm{H}_{2}$ production).

\section{Expression and Regulation of the Genes Involved in Hydrogen Production}

In order to use cyanobacteria for the biological production of hydrogen, it is important to thoroughly study the regulation of the hydrogen-production machine in order to better understand its role in the global cell metabolism (Figure 2) and identify bottlenecks limiting $\mathrm{H}_{2}$ production.

In contrast to the hypABCDEF genes that are scattered in the Synechocystis chromosome (Figure 1), the hoxEFUYH genes are clustered in an octacistronic operon, which comprises the hoxE, hoxF, sll1222, hox $U$, hoxY, ssl2420, sll1225 and hoxH genes in that order, and also encode three proteins of unknown function: S111222, Ss12420 and S111225 [14,20]. This operon is weakly expressed as a polycistronic transcript, which initiates 168 bp upstream of the start codon of the hoxE gene [22,23]. The promoter of the hoxEFUYH operon is not very active [16] though it harbors the two sequences resembling canonical E.coli-like promoter boxes, -35 (TTGctc) and -10 (TAacAa) located at correct distances from each other $(18 \mathrm{bp})$ and from the transcription start site $(7 \mathrm{bp})$. As such canonical promoters were long ago shown to be strongly active in Synechocystis [24], this indicated that it is negatively regulated (see below).

The hoxEFUYH operon is regulated by various environmental conditions, such as hydrogen, light, nitrate, nickel, oxygen and sulfur availabilities [25], in agreement with the finding that $\mathrm{H}_{2}$ production can be increased in media with optimized concentrations of these elements [26]. The transcript levels of all hox genes are increased (five to sixfold) under microaerobic conditions [27,28], with an additional induction (10-12-fold) of hoxEF in darkness [27].

The upregulation of hox genes elicited by nitrogen starvation is consistent with the increased $\mathrm{H}_{2}$ production triggered by inhibition of nitrate assimilation, suggesting that hydrogenase serves as an alternative sink for photosynthetic electrons no longer used for nitrate reduction [29].

The hox genes are also regulated by inhibitors of the photosynthetic electron transport chain in a way suggesting that the redox state of the plastoquinone pool does not play a prominent role in hox regulation [27] and $\mathrm{H}_{2}$ production [26]. Furthermore, the expression of the hoxEFUYH operon is controlled by three transcription factors (Figure 2); two positively acting regulators, LexA (S111626) [22,23] and AbrB1 (S110359) [30], and one repressor AbrB2 (S110822) [16]. 
Figure 2. Schematic representation of the redox (electron transfer) metabolism of Synechocystis growing in photoautotrophic conditions. The electron transfers and transcriptional regulations are represented by solid black arrows and dotted blue arrows, respectively. The redox dependent- and metal requiring-processes are indicated by orange and red dotted arrows, respectively. PSII, photosystem II; PSI, photosystem I, Cytb6/f, cytochrome b6/f; Fed, ferredoxin; Flv, flavodiiron protein; FNR, ferredoxin NADP reductase; FTR, ferredoxin thioredoxin reductase; GltS, ferredoxin dependent glutamate synthase; NarB, Nitrate reductase; NirA, Nitrite reductase; NDH1, NADPH oxidoreductase complex 1; NDH2, NADPH oxidoreductase type 2; PQ, plastoquinone pool; SDH, succinate dehydrogenase; Sir, sulfite reductase; $\operatorname{Trx}=$ Thioredoxin.

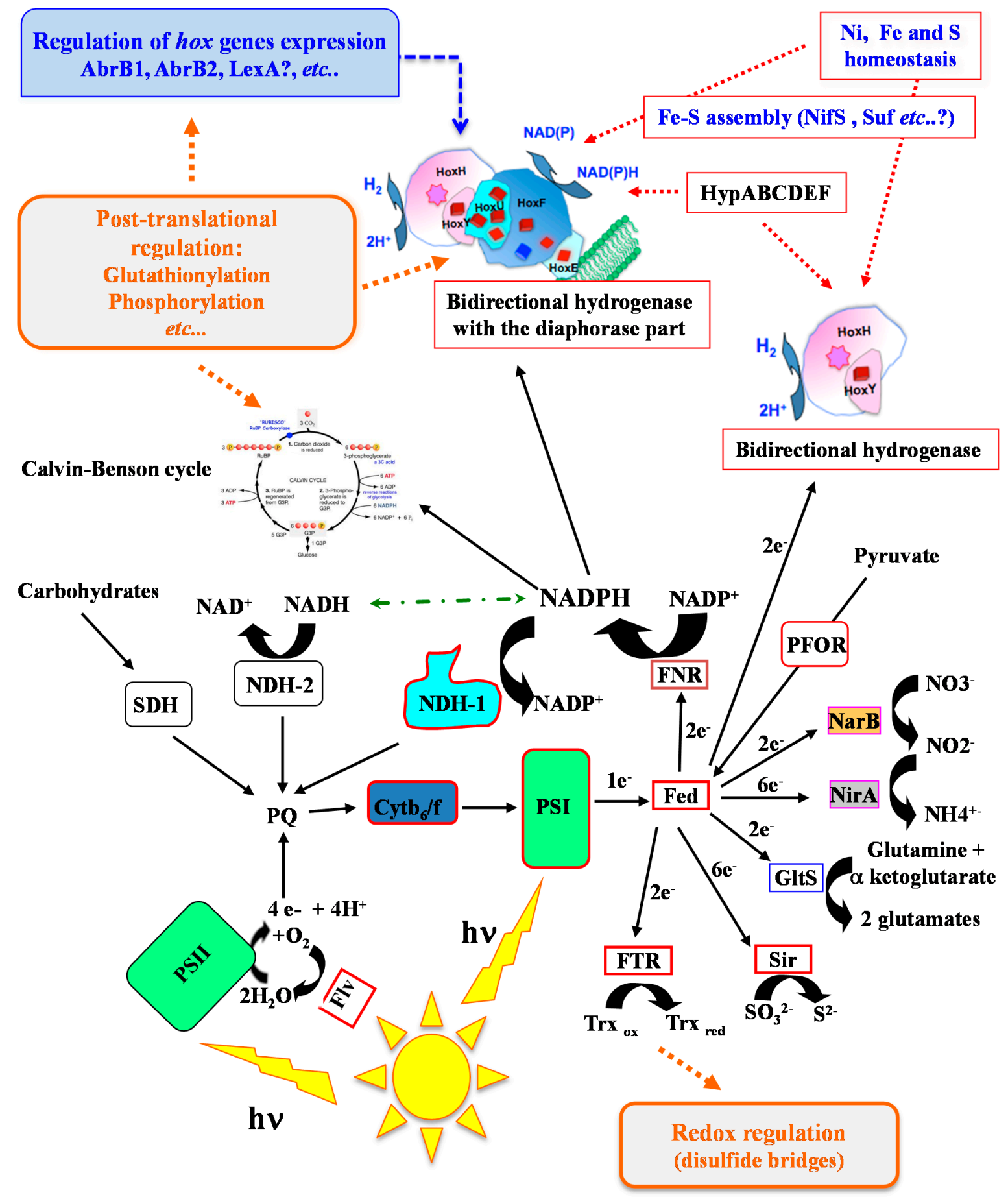




\subsection{LexA a Positively Acting Player in Hydrogen Production}

By using streptavidin-coated magnetic beads in DNA affinity assays two groups identified the LexA protein as capable of binding to two regions of the hox promoter region, i.e., at 198-338 bp [23] or 592-690 bp [22] upstream of the hoxE start codon. Prior to these studies, the LexA regulator was found not to regulate typical DNA repair genes, unlike what occurs in E. coli, but instead to control genes involved in carbon metabolism [31] and the redox-responsive gene $c r h R$ encoding an RNA helicase involved in translational regulation [32]. Furthermore, proteomic analyses identified LexA as protein associated to plasma membrane and thylakoids [25].

The occurrence of two LexA binding sites in the hox promoter suggest that the LexA-mediated regulation involves DNA bending [14], a well-known phenomenon in transcriptional regulation. Furthermore, the depletion of the LexA protein decreased the hydrogenase activity, suggesting that LexA plays a positive role in hydrogen production [25]. To test the influence of LexA on the transcription of the hox operon, we cloned the hox promoter in front of the promoter-less cat reporter gene of the promoter-probe plasmid vector pSB2A, which replicates stably in Synechocystis at one copy per chromosome copy [9]. Then, we introduced the corresponding recombinant plasmid pSB2A-hox promoter in the WT (wild-type) strain and $\Delta l e x A$ mutants where we measured the expression of the cat (chloramphenicol acetyl transferase) gene driven by the hox promoter. Similar CAT activities were obtained in the WT and $\triangle l e x A$ strains growing in standard photoautotrophic conditions, indicating that the activity of the hox promoter is not affected by the absence of LexA (data not shown). This finding challenges the notion that LexA is a direct transcription regulator of the hox operon.

\subsection{The AbrB1 Protein Positively Regulates the Transcription of the hoxEFUHY Operon}

By using DNA affinity assays, another transcription factor positively acting on hox expression was isolated on the basis of its specific interaction with the hox promoter region. This regulator, AbrB1, resembles the AbrB regulator of $B$. subtilis, which regulates about 100 genes involved in various processes (sporulation, biofilm formation, antibiotic production, development of competence for DNA uptake), though the cyanobacterial AbrB1 protein has its putative DNA-binding domain in its $C$-terminal region, instead of in the usual $N$-terminal region as occurs in non-cyanobacterial AbrB proteins [30,33]. The Synechocystis AbrB1 protein was found to be indispensable to cell life in both the wild-type strain [30] and the glucose tolerant mutant [33]. Interestingly, AbrB1 was found to interact with a high degree of confidence with itself, suggesting that AbrB1 acts as an oligomer [25]. The abrBl gene is expressed from an atypical promoter harboring an extended -10 element (5'-tgtTATAtT-3') [30], which might compensate the absence of a genuine -35 box (5'-TTGACA-3'; located at the correct $17 \pm 1 \mathrm{bp}$ distance from the -10 element) similarly to what found for the $\sec A$ gene [34].

\subsection{The AbrB2 Repressor of the hoxEFUYH Operon Plays a Prominent Role in the Regulation and the} Tolerance to Oxidative and Metal Stresses

Because it resembles to AbrB1, the AbrB2 has also been studied in Synechocystis, and appeared to be dispensable to the growth of both the glucose-tolerant mutant [33] and the wild-type strain [16]. 
In the glucose-tolerant mutant, AbrB2 appeared to regulate numerous genes involved in nitrogen and carbon assimilation, and to interact with AbrB1 [33,35,36]. In the wild-type strain AbrB2 was shown to repress its own gene and the hox operon [16]. The others genes directly involved in hydrogen production: hox $W$, hyp, lexA and $a b r B 1$ were not regulated by AbrB2, even though it appeared to regulate a large number of genes (about 330 genes, mostly negatively), as shown by transcriptome analysis with pan-genomic microarrays [37]. The highest number of the AbrB2-responsive genes code for hypothetical proteins or unknown proteins (about 200 genes), emphasizing that we still have a limited knowledge of Synechocystis, though it is one of the more intensively studied cyanobacterium. Many these unknown genes belong to the endogenous plasmids, suggesting that they play a role in hydrogen production.

The AbrB2 master regulator was also found to regulate several transport genes, including cysAPUW $\left(\mathrm{SO}_{4}{ }^{2-}\right)$, fecBCD $\left(\mathrm{Fe}^{2+}\right)$, in agreement with the Hox enzyme using a Ni-Fe and several $\mathrm{Fe}-\mathrm{S}$ redox clusters to produce hydrogen [37]. In this $a b r B 2$-deleted mutant, the increased expression of the anti-oxidant genes $c y d A B$ (cytochrome bd-quinol oxidase that can reduce $\mathrm{O}_{2}$ ) and nor $B$ (nitric oxide reductase) are consistent with the increased production of hydrogen [16], which is regarded as an anti-oxidant process that evacuates electrons occurring in excess to prevent them to reduce molecular oxygen and produce toxic reactive oxygen species $[12,15,17,18]$. Consistently, the absence of the AbrB2 repressor led to an increased tolerance of Synechocystis to both metal and oxidative stresses triggered by nickel and the thiol oxidizing agent diamide [38].

The $a b r B 2$ promoter region was also analyzed, using transcriptional fusion to the promoter-less cat reporter gene of the plasmid pSB2A that replicates stably in Synechocystis at one copy per chromosome copy [9]. The abrB2 gene was found to expressed from an atypical promoter, which harbors an extended -10 element (5'-TGTATAAT-3'), likely compensating the absence of a genuine -35 box (5'-TTGACA-3'; located at about $17 \pm 1$ bp from the -10 element), similarly to the genes sec $A$ [34], gap2 [39] and $a b r B 1$ [30]. In addition, a consensus DNA motif, TT-( $\left.\mathrm{N}_{5}\right)-\mathrm{AAC}$, was also proposed for AbrB2 binding [16].

\section{Function and Over-Production of the Hydrogen Production Machine}

To attempt increasing hydrogenase activity in Synechocystis, Germer and co-workers used the light-inducible promoter of the photosynthetic gene $p s b A I I$ to increase the expression of the endogenous hoxEFUYH operon and the heterologous NoshypABCDEF operon from Nostoc PCC7120 [40]. The gain in activity was modest (3.2-fold; i.e., from $2.9 \mathrm{nmol} \mathrm{H} \cdot \mathrm{min}^{-1} \cdot \mathrm{mg} \cdot \mathrm{chlorophyll}^{-1}$ in wild-type cells up to $9.4 \mathrm{nmol} \mathrm{H} 2 \cdot \mathrm{min}^{-1} \cdot \mathrm{mg} \cdot$ chlorophyll ${ }^{-1}$ in mutant cells) for three main possible reasons. First, the light-inducible psbAII promoter used to increase the expression of the hoxEFUYH and the Noshyp $A B C D E F$ genes is more active under high light that increases the photosynthetic production of $\mathrm{O}_{2}$, which inhibits hydrogenase activity. Second, the Nostoc HypABCDEF proteins might be not fully active on the Synechocystis PCC6803 HoxEFUYH proteins. Third, it is also possible that the Nostoc HypABCDEF proteins might somehow interfere with the function of the endogenous Synechocystis HypABCDEF proteins.

To increase the expression of the hoxEFUYH operon and the hypABCDEF genes, we have used the strong $\lambda p_{R}$ promoter $[10,24]$. Practically, we replaced the weak [16] natural promoter of the hoxEFUYH 
operon by the $\lambda p_{R}$ promoter, and we cloned the hypABCDEF genes under the control of the same $\lambda p_{R}$ promoter, in a plasmid that replicates at the same 10-20 copies per cell as the chromosome [10]. The mutant grew as fit as the WT strain. It strongly over-expressed the hoxEFUYH operon and the hyp $A B C D E F$ genes (about 100-fold), and exhibited a 20-fold higher levels of active hydrogenase than the WT strain. These findings show that an increased production of active hydrogenase is not detrimental to cell life, and other factors than hoxEFUYH and hypABCDEF transcription limit hydrogen production (see below).

\section{Role of the Hydrogen Production Machine}

Though, the complex hydrogenase enzyme is neither ubiquitous in all cyanobacteria nor essential to the standard photoautotrophic growth of model strains [12], the analysis of gene deletion and overexpression mutants showed that the presence of the Hox enzyme offers some growth advantages in certain environmental situations.

The hoxEFUYH Operon Operates in the Protection against Redox Stresses Triggered by Hydrogen Peroxide or the Reduced Carbon Metabolites Glucose and Glycerol

All aerobic organisms invariably produce reactive oxygen species, such as $\mathrm{H}_{2} \mathrm{O}_{2}$, through the accidental autoxidation of redox enzymes [41], which occurs when their reduced cofactors reduce oxygen. This phenomenon is important in cyanobacteria, because their active photosynthesis massively produces oxygen and electrons [42]. As the cyanobacterial hydrogenase enzyme complex has been proposed to act as an electron valve releasing some of the supernumerary electrons [12,17], we have compared the $\mathrm{H}_{2} \mathrm{O}_{2}$ tolerance of the WT strain to that of mutants which either lack the hoxEFUYH operon, or overexpress it, alone or in combination with the hypABCDEF genes [15]. The data showed that the hoxEFUYH operon and the hypABCDEF genes contribute to the protection against $\mathrm{H}_{2} \mathrm{O}_{2}$, positively (hoxEFUYH operon) or negatively (hyp $A B C D E F)$. Future experiments will be required to test whether the higher $\mathrm{H}_{2} \mathrm{O}_{2}$ tolerance directed by the overexpression of the hoxEFUYH operon is due to the increased abundance of (i) the HoxHY hydrogenase enzyme per se; (ii) the HoxEFU diaphorase enzyme; and/or (iii) the S111222, Ss12420 and S111225 proteins of as yet unknown function.

In addition, we have tested the influence on hydrogenase deletion or overproduction mutants of the reduced-carbon metabolites glucose and glycerol, a cheap surplus of industries [43] that stimulate hydrogen production in the cyanobacteria Arthrospira (Spirulina) maxima [44] and Cyanothece ATCC 51142 [45]. Both glucose and glycerol might be toxic to Synechocystis growing under an otherwise normal light fluence, probably because these reduced metabolites somehow decrease the electrons-consuming $\mathrm{CO}_{2}$-assimilation, thereby allowing spared electrons to recombine with $\mathrm{O}_{2}$ and generate ROS [46]. The level of tolerance to these stresses increased in parallel with the abundance of the Hox proteins [15], in agreement with the higher tolerance to a mixture of both glucose and arginine of the WT strain as compared to a hoxH-deletion mutant [18]. Collectively, these data are consistent with the proposal that hydrogenase operates as an electron valve preventing supernumerary electrons generated by photosynthesis and/or sugar catabolism to recombine with $\mathrm{O}_{2}$ to generate toxic reactive oxygen species [12]. 


\section{Glutathionylation a Probable New Player in Hydrogen Production}

As mentioned above, cyanobacteria are continuously challenged with toxic reactive oxygen species generated by photosynthesis and respiration, which can oxidize the thiol group (SH) of two cysteinyl residues to form disulfide bonds (-S-S-) between proteins, or between a protein and a molecule of the, crucial [47], anti-oxidant tripeptide glutathione (glutathione-protein mixed disulfide, also termed glutathionylation) $[41,42]$. Collectively, the data suggesting that the hox operon repressor AbrB2 exists under two uncharacterized posttranslational modification forms [35,48], and the findings that AbrB2 and the hox operon play a role in the tolerance to oxidative stresses [15,38], prompted us to test whether these proteins might be glutathionylated. We found that the single, widely conserved cysteine of AbrB2 is the target of glutathionylation, which affects the oligomerization of AbrB2; its binding on the hox operon-promoter DNA; its repression of a wealth of other genes; and its stability at the non-standard temperature of $39{ }^{\circ} \mathrm{C}$ [38]. The role of AbrB2 can be viewed as follows. In cells growing in absence of stress, AbrB2 down-regulates hydrogen production and other stress defenses. By contrast, in cells facing oxidative stresses triggered by light excess or metal availabilities, AbrB2 is oxidized and its single cysteine is glutathionylated. Thus, AbrB2 is no longer able to repress hydrogen production thereby allowing evacuation of excess electrons, among other anti-oxidant processes. After recovery from oxidative stress, AbrB2 activity is restored (likely through deglutathionylation catalyzed by glutaredoxins [49]) and starts repressing hydrogen production and other anti-oxidant process, to save photosynthetic electrons. Similarly, we found that the cysteine of the other hoxEFUYH regulator, AbrB1, which interacts with AbrB2 and also regulates the hoxEFUYH operon [30,33,36], can be glutathionylated in vitro like AbrB2. Thus, it will be interesting in the future to analyze the influence of the glutathionylation of $\mathrm{AbrB} 1$ and $\mathrm{AbrB} 2$ on their interaction and their regulation of hydrogen production. To our knowledge, AbrB2 and AbrB1 are the first cyanobacterial regulators reported to undergo glutathionylation (in other prokaryotes, only the OxyR regulator is known to be controlled by glutathionylation [50]), emphasizing on the evolutionary conservation of this process, well described in eukaryotes [51].

Stimulated by the findings that both AbrB1 and AbrB2, and the mercuric reductase enzyme [49], can be glutathionylated, we performed a large-scale proteomic analysis of glutathionylation [52]. Amongst the glutathionylatable proteins, we found HoxH and HoxF. Interestingly, the glutathionylated Cys (C289) of HoxF is located close to the putative binding site for NAD(P). These findings will certainly stimulate the analysis of the crosstalk between hydrogen production and the oxidative stress-responsive glutathionylation-and-deglutathionylation process.

\section{Conclusions}

Though significant progress has been made recently in our understanding of the function and regulation of the complex hydrogen $\mathrm{Ni}-\mathrm{Fe}$ hydrogenase of the model cyanobacterium Synechocystis PCC6803, important questions remain.

Recent in vitro data suggested that the Hox enzyme can receive electrons from not only $\mathrm{NAD}(\mathrm{P}) \mathrm{H}$ as usually shown, but also, or even preferentially, from ferredoxin. This proposal will certainly stimulate 
the investigation of the selectivity/redundancy of the nine ferredoxins of Synechocystis, which are conserved in cyanobacteria.

Using gene deletion and over-expression, it has been shown recently that the hoxEFUYH operon operates in the defense against redox stresses triggered by $\mathrm{H}_{2} \mathrm{O}_{2}$ or reduced carbon or nitrogen metabolites. These findings strengthen the proposal that hydrogenase operates as an electron valve to prevent the supernumerary electrons to recombine with $\mathrm{O}_{2}$ to generate toxic reactive oxygen species. Hence, the hydrogenase complex can be viewed as an important enzyme in cyanobacteria like Synechocystis, which can grow in biofilm, a thick network of auto-aggregated cells where they are inevitably exposed to $\mathrm{H}_{2} \mathrm{O}_{2}$ and reduced metabolites released by their neighbors (living or dying and lysing). This view is strengthened by the absence of hydrogenase enzyme in most planktonic cyanobacteria living in open oceans. It will be interesting in the future to investigate the influence of the Synechocystis hydrogenase enzyme in the growth and response to environmental stresses of cells incubated not only in agitated liquid cultures but also in static conditions or on solid media to favor the formation of biofilm.

The recently achieved simultaneous overproduction of the HoxEFUYH and HypABCDEF proteins within the same cells led to a 20-fold increase in active hydrogenase. These sophisticated mutants with a higher hydrogenase content and a healthy growth will be very useful cell factories for the purification of large hydrogenase quantities for structural analyses, the data of which should enable the design of a meaningful mutational strategy to increase the low natural $\mathrm{O}_{2}$ tolerance of the hydrogenase enzyme to increase hydrogen production.

All hydrogenase-overproducing mutants displayed higher levels of expression of the hoxEFUYH and hyp $A B C D E F$ genes than that of active hydrogenase, indicating that limiting post-transcriptional factors should be dealt with. Thus, it will be important to pay particular attention to glutathionylation (the formation of a mixed-disulfide between the cysteines residues of a protein and the cysteine residue of glutathione) because it was recently reported that the AbrB1 and AbrB2 hydrogen regulator and the HoxH (diaphorase) and HoxF (hydrogenase) protein-subunits can be glutathionylated. These findings will certainly stimulate the analysis of the redox crosstalk between hydrogen production and the oxidative stress-responsive glutathionylation-and-deglutathionylation process.

\section{Acknowledgments}

This work was supported in part by the French scientific Program "ANR-09-BIOE-002-01 (EngineeringH2cyano)". Théo Veaudor is recipient of a fellowship (IRTELIS) from the CEA (France).

\section{Author Contributions}

Thorough analysis of the literature: Corinne Cassier-Chauvat, Franck Chauvat and Théo Veaudor. Writing of the paper: Corinne Cassier-Chauvat, Franck Chauvat and Théo Veaudor.

\section{Conflicts of Interest}

The authors declare no conflict of interest. 


\section{References}

1. Armaroli, N.; Balzani, V. The hydrogen issue. ChemSusChem 2011, 4, 21-36.

2. Zhou, J.; Li, Y. Engineering cyanobacteria for fuels and chemicals production. Protein Cell 2010, 1, 207-210.

3. Rosgaard, L.; de Porcellinis, A.J.; Jacobsen, J.H.; Frigaard, N.U.; Sakuragi, Y. Bioengineering of carbon fixation, biofuels, and biochemicals in cyanobacteria and plants. J. Biotechnol. 2012, 162, 134-147.

4. Dismukes, G.C.; Carrieri, D.; Bennette, N.; Ananyev, G.M.; Posewitz, M.C. Aquatic phototrophs: Efficient alternatives to land-based crops for biofuels. Curr. Opin. Biotechnol. 2008, 19, 235-240.

5. Ducat, D.C.; Way, J.C.; Silver, P.A. Engineering cyanobacteria to generate high-value products. Trends Biotechnol. 2011, 29, 95-103.

6. Zehr, J.P. Nitrogen fixation by marine cyanobacteria. Trends Microbiol. 2011, 19, 162-173.

7. Jansson, C.; Northen, T. Calcifying cyanobacteria-The potential of biomineralization for carbon capture and storage. Curr. Opin. Biotechnol. 2010, 21, 365-371.

8. Kruse, O.; Rupprecht, J.; Mussgnug, J.H.; Dismukes, G.C.; Hankamer, B. Photosynthesis: A blueprint for solar energy capture and biohydrogen production technologies. Photochem. Photobiol. Sci. 2005, 4, 957-970.

9. Marraccini, P.; Bulteau, S.; Cassier-Chauvat, C.; Mermet-Bouvier, P.; Chauvat, F. A conjugative plasmid vector for promoter analysis in several cyanobacteria of the genera Synechococcus and Synechocystis. Plant Mol. Biol. 1993, 23, 905-909.

10. Mermet-Bouvier, P.; Chauvat, F. A conditional expression vector for the cyanobacteria Synechocystis sp. strains PCC6803 and PCC6714 or Synechococcus sp. strains PCC7942 and PCC6301. Curr. Microbiol. 1994, 28, 145-148.

11. Huang, H.H.; Camsund, D.; Lindblad, P.; Heidorn, T. Design and characterization of molecular tools for a Synthetic Biology approach towards developing cyanobacterial biotechnology. Nucleic Acids Res. 2010, 38, 2577-2593.

12. Carrieri, D.; Wawrousek, K.; Eckert, C.; Yu, J.; Maness, P.C. The role of the bidirectional hydrogenase in cyanobacteria. Bioresour. Technol. 2011, 102, 8368-8377.

13. Bothe, H.; Schmitz, O.; Yates, M.G.; Newton, W.E. Nitrogen fixation and hydrogen metabolism in cyanobacteria. Microbiol. Mol. Biol. Rev. 2010, 74, 529-551.

14. Tamagnini, P.; Leitao, E.; Oliveira, P.; Ferreira, D.; Pinto, F.; Harris, D.J.; Heidorn, T.; Lindblad, P. Cyanobacterial hydrogenases: Diversity, regulation and applications. FEMS Microbiol. Rev. 2007, 31, 692-720.

15. Ortega-Ramos, M.; Jittawuttipoka, T.; Saenkham, P.; Czarnecka-Kwasiborski, A.; Bottin, H.; Cassier-Chauvat, C.; Chauvat, F. Engineering Synechocystis PCC6803 for hydrogen production: influence on the tolerance to oxidative and sugar stresses. PLoS One 2014, 9, e89372.

16. Dutheil, J.; Saenkham, P.; Sakr, S.; Leplat, C.; Ortega-Ramos, M.; Bottin, H.; Cournac, L.; Cassier-Chauvat, C.; Chauvat, F. The AbrB2 autorepressor, expressed from an atypical promoter, represses the hydrogenase operon to regulate hydrogen production in Synechocystis strain PCC6803. J. Bacteriol. 2012, 194, 5423-5433. 
17. Cournac, L.; Guedeney, G.; Peltier, G.; Vignais, P.M. Sustained photoevolution of molecular hydrogen in a mutant of Synechocystis sp. strain PCC 6803 deficient in the type I NADPH-dehydrogenase complex. J. Bacteriol. 2004, 186, 1737-1746.

18. Gutekunst, K.; Chen, X.; Schreiber, K.; Kaspar, U.; Makam, S.; Appel, J. The bidirectional NiFe-hydrogenase in Synechocystis sp. PCC 6803 is reduced by flavodoxin and ferredoxin and is essential under mixotrophic, nitrate-limiting conditions. J. Biol. Chem. 2014, 289, 1930-1937.

19. Ghirardi, M.L.; Dubini, A.; Yu, J.; Maness, P.C. Photobiological hydrogen-producing systems. Chem. Soc. Rev. 2009, 38, 52-61.

20. Eckert, C.; Boehm, M.; Carrieri, D.; Yu, J.; Dubini, A.; Nixon, P.J.; Maness, P.C. Genetic Analysis of the Hox Hydrogenase in the Cyanobacterium Synechocystis sp. PCC 6803 Reveals Subunit Roles in Association, Assembly, Maturation, and Function. J. Biol. Chem. 2012, 287, 43502-43515.

21. McIntosh, C.L.; Germer, F.; Schulz, R.; Appel, J.; Jones, A.K. The [NiFe]-hydrogenase of the cyanobacterium Synechocystis sp. PCC 6803 works bidirectionally with a bias to $\mathrm{H}_{2}$ production. J. Am. Chem. Soc. 2011, 133, 11308-11319.

22. Gutekunst, K.; Phunpruch, S.; Schwarz, C.; Schuchardt, S.; Schulz-Friedrich, R.; Appel, J. LexA regulates the bidirectional hydrogenase in the cyanobacterium Synechocystis sp. PCC 6803 as a transcription activator. Mol. Microbiol. 2005, 58, 810-823.

23. Oliveira, P.; Lindblad, P. LexA, a transcription regulator binding in the promoter region of the bidirectional hydrogenase in the cyanobacterium Synechocystis sp. PCC 6803. FEMS Microbiol. Lett. 2005, 251, 59-66.

24. Ferino, F.; Chauvat, F. A promoter-probe vector-host system for the cyanobacterium, Synechocystis PCC6803. Gene 1989, 84, 257-266.

25. Oliveira, P.; Lindblad, P. Transcriptional regulation of the cyanobacterial bidirectional Hox-hydrogenase. Dalton Trans. 2009, 45, 9990-9996.

26. Burrows, E.H.; Chaplen, F.W.; Ely, R.L. Effects of selected electron transport chain inhibitors on 24-h hydrogen production by Synechocystis sp. PCC 6803. Bioresour. Technol. 2011, 102, 3062-3070.

27. Kiss, E.; Kos, P.B.; Vass, I. Transcriptional regulation of the bidirectional hydrogenase in the cyanobacterium Synechocystis 6803. J. Biotechnol. 2009, 142, 31-37.

28. Summerfield, T.C.; Toepel, J.; Sherman, L.A. Low-oxygen induction of normally cryptic $p s b A$ genes in cyanobacteria. Biochemistry 2008, 47, 12939-12941.

29. Krasikov, V.; Aguirre von Wobeser, E.; Dekker, H.L.; Huisman, J.; Matthijs, H.C. Time-series resolution of gradual nitrogen starvation and its impact on photosynthesis in the cyanobacterium Synechocystis PCC 6803. Physiol. Plant 2012, 145, 426-439.

30. Oliveira, P.; Lindblad, P. An AbrB-Like protein regulates the expression of the bidirectional hydrogenase in Synechocystis sp. strain PCC 6803. J. Bacteriol. 2008, 190, 1011-1019.

31. Domain, F.; Houot, L.; Chauvat, F.; Cassier-Chauvat, C. Function and regulation of the cyanobacterial genes $l e x A, \operatorname{rec} A$ and $\operatorname{ruv} B$ : LexA is critical to the survival of cells facing inorganic carbon starvation. Mol. Microbiol. 2004, 53, 65-80. 
32. Patterson-Fortin, L.M.; Colvin, K.R.; Owttrim, G.W. A LexA-related protein regulates redox-sensitive expression of the cyanobacterial RNA helicase, crhR. Nucleic Acids Res. 2006, $34,3446-3454$.

33. Ishii, A.; Hihara, Y. An AbrB-like transcriptional regulator, Sll0822, is essential for the activation of nitrogen-regulated genes in Synechocystis sp. PCC 6803. Plant Physiol. 2008, 148, 660-670.

34. Mazouni, K.; Bulteau, S.; Cassier-Chauvat, C.; Chauvat, F. Promoter element spacing controls basal expression and light inducibility of the cyanobacterial secA gene. Mol. Microbiol. 1998, 30, 1113-1122.

35. Lieman-Hurwitz, J.; Haimovich, M.; Shalev-Malul, G.; Ishii, A.; Hihara, Y.; Gaathon, A.; Lebendiker, M.; Kaplan, A. A cyanobacterial AbrB-like protein affects the apparent photosynthetic affinity for $\mathrm{CO}_{2}$ by modulating low- $\mathrm{CO}_{2}$-induced gene expression. Environ. Microbiol. 2009, 11, 927-936.

36. Yamauchi, Y.; Kaniya, Y.; Kaneko, Y.; Hihara, Y. Physiological roles of the cyAbrB transcriptional regulator pair S110822 and S110359 in Synechocystis sp. strain PCC 6803. J. Bacteriol. 2011, 193, 3702-3709.

37. Leplat, C.; Champeimont, R.; Saenkham, P.; Cassier-Chauvat, C.; Aude, J.-C.; Chauvat, F. Genome-wide transcriptome analysis of hydrogen production in the cyanobacterium Synechocystis: Towards the identification of new players. Int. J. Hydrog. Energ. 2013, 38, 1866-1872.

38. Sakr, S.; Dutheil, J.; Saenkham, P.; Bottin, H.; Leplat, C.; Ortega-Ramos, M.; Aude, J.; Chapuis, V.; Guedeney, G.; Decottignies, P.; et al. The activity of the Synechocystis PCC6803 AbrB2 regulator of hydrogen production can be post-translationally controlled through glutathionylation. Int. J. Hydrog. Energ. 2013, 38, 13547-13555.

39. Figge, R.M.; Cassier-Chauvat, C.; Chauvat, F.; Cerff, R. The carbon metabolism-controlled Synechocystis gap2 gene harbours a conserved enhancer element and a Gram-positive-like -16 promoter box retained in some chloroplast genes. Mol. Microbiol. 2000, 36, 44-54.

40. Germer, F.; Zebger, I.; Saggu, M.; Lendzian, F.; Schulz, R.; Appel, J. Overexpression, isolation, and spectroscopic characterization of the bidirectional [NiFe] hydrogenase from Synechocystis sp. PCC 6803. J. Biol. Chem. 2009, 284, 36462-36472.

41. Imlay, J.A. The molecular mechanisms and physiological consequences of oxidative stress: Lessons from a model bacterium. Nat. Rev. Microbiol. 2013, 11, 443-454.

42. Narainsamy, K.; Marteyn, B.; Sakr, S.; Cassier-Chauvat, C.; Chauvat, F. Genomics of the pleïotropic glutathione system in cyanobacteria. In Advances in Botanical Research; Chauvat, F., Cassier-Chauvat, C., Eds.; Academic Press, Elsevier: Amsterdam, The Netherlands, 2013; Volume 65, pp. 157-188.

43. Da Silva, G.P.; Mack, M.; Contiero, J. Glycerol: A promising and abundant carbon source for industrial microbiology. Biotechnol. Adv. 2009, 27, 30-39.

44. Carrieri, D.; Ananyev, G.; Costas, A.M.G.; Bryant, D.A.; Dismukes, G.C. Renewable hydrogen production by cyanobacteria: Nickel requirements for optimal hydrogenase activity. Int. J. Hydrog. Energ. 2008, 33, 2014-2022.

45. Bandyopadhyay, A.; Stockel, J.; Min, H.; Sherman, L.A.; Pakrasi, H.B. High rates of photobiological $\mathrm{H}_{2}$ production by a cyanobacterium under aerobic conditions. Nat. Commun. 2010, 1, doi:10.1038/ncomms1139. 
46. Narainsamy, K.; Cassier-Chauvat, C.; Junot, C.; Chauvat, F. High performance analysis of the cyanobacterial metabolism via liquid chromatography coupled to a LTQ-Orbitrap mass spectrometer: Evidence that glucose reprograms the whole carbon metabolism and triggers oxidative stress. Metabolomics 2013, 9, 21-32.

47. Cameron, J.C.; Pakrasi, H.B. Essential role of glutathione in acclimation to environmental and redox perturbations in the cyanobacterium Synechocystis sp. PCC 6803. Plant Physiol. 2010, 154, $1672-1685$.

48. Sazuka, T.; Yamaguchi, M.; Ohara, O. Cyano2Dbase updated: Linkage of 234 protein spots to corresponding genes through $N$-terminal microsequencing. Electrophoresis 1999, 20, 2160-2171.

49. Marteyn, B.; Sakr, S.; Farci, S.; Bedhomme, M.; Chardonnet, S.; Decottignies, P.; Lemaire, S.D.; Cassier-Chauvat, C.; Chauvat, F. The Synechocystis PCC6803 MerA-like enzyme operates in the reduction of both mercury and uranium, under the control of the glutaredoxin 1 enzyme. J. Bacteriol. 2013, 195, doi:10.1128/JB.00272-13.

50. Masip, L.; Veeravalli, K.; Georgiou, G. The many faces of glutathione in bacteria. Antioxid. Redox Signal. 2006, 8, 753-762.

51. Zaffagnini, M.; Bedhomme, M.; Marchand, C.H.; Morisse, S.; Trost, P.; Lemaire, S.D. Redox regulation in photosynthetic organisms: Focus on glutathionylation. Antioxid. Redox Signal. 2012, 16, 567-586.

52. Chardonnet, S.; Sakr, S.; Cassier-Chauvat, C.; Le Maréchal, P.; Chauvat, F.; Lemaire, S.; Decottignies, P. First proteomic study of S-glutathionylation in cyanobacteria. J. Proteome Res. 2014, doi:10.1021/pr500625a.

(C) 2014 by the authors; licensee MDPI, Basel, Switzerland. This article is an open access article distributed under the terms and conditions of the Creative Commons Attribution license (http://creativecommons.org/licenses/by/4.0/). 